\begin{tabular}{|c|c|}
\hline Title & $\begin{array}{l}\text { Post-relapse survival in patients with the early and late distant recurrence in estrogen receptor-positive HER2-negative } \\
\text { breast cancer }\end{array}$ \\
\hline Author(s) & $\begin{array}{l}\text { Ogiya, A kiko; Y amazaki, Kieko; Horii, Rie; Shien, T adahiko; Horimoto, Y oshiya; Masuda, Norikazu; Inao, Touko; } \\
\text { Hosoda, Mitsuchika; I shida, Naoko; Osako, T omofumi; Takahashi, Masato; Endo, Y umi; Miyoshi, Y uichiro; Y asojima, } \\
\text { Hiroyuki; T omioka, Nobumoto; Y amashita, Hiroko; Collaborative Study Group of Scientific Research of the Japanese } \\
\text { Breast Cancer Society }\end{array}$ \\
\hline Citation & $\begin{array}{l}\text { Breast cancer, } 24(3), 473-482 \\
\text { https://doi.org/10.1007/s12282-016-0730-3 }\end{array}$ \\
\hline Issue Date & 2017-05 \\
\hline Doc URL & http:/hdl.handle.net/2115/70047 \\
\hline Rights & The final publication is available at link.springer.com \\
\hline Type & article (author version) \\
\hline File Information & BreastCancer24_473.pdf \\
\hline
\end{tabular}

Instructions for use 


\section{Post-relapse survival in patients with early and late distant recurrence in estrogen receptor-positive, HER2-negative breast cancer}

Akiko Ogiya $^{1}$, Kieko Yamazaki ${ }^{1}$, Rie Horii ${ }^{2}$, Tadahiko Shien ${ }^{3}$, Yoshiya Horimoto ${ }^{4}$, Norikazu Masuda $^{5}$, Touko Inao ${ }^{6}$, Mitsuchika Hosoda ${ }^{7}$, Naoko Ishida ${ }^{7}$, Tomofumi Osako ${ }^{8}$, Masato Takahashi $^{9}$, Yumi Endo ${ }^{10}$, Yuichiro Miyoshi ${ }^{3}$, Hiroyuki Yasojima ${ }^{5}$, Nobumoto Tomioka ${ }^{9}$, Hiroko Yamashita ${ }^{7 *}$; Collaborative Study Group of Scientific Research of the Japanese Breast Cancer Society

1 Breast Surgical Oncology, Cancer Institute Hospital, Japanese Foundation for Cancer Research, Tokyo, Japan

${ }^{2}$ Division of Pathology, Cancer Institute of the Japanese Foundation for Cancer Research, Tokyo, Japan

${ }^{3}$ Breast and Endocrine Surgery, Okayama University Hospital, Okayama, Japan

${ }^{4}$ Department of Breast Oncology, Juntendo University School of Medicine, Tokyo, Japan

${ }^{5}$ Department of Surgery, Breast oncology, NHO Osaka National Hospital, Osaka, Japan

6 Department of Breast \& Endocrine Surgery, Graduate School of Medical Science Kumamoto University, Kumamoto, Japan

${ }^{7}$ Breast Surgery, Hokkaido University Hospital, Sapporo, Japan

${ }^{8}$ Department of Breast and Endocrine Surgery, Kumamoto City Hospital, Kumamoto, Japan $\dagger$ $†$ Present address: Kumamoto Shinto General Hospital, Kumamoto, Japan

${ }^{9}$ Department of Breast Surgery, NHO Hokkaido Cancer Center, Sapporo, Japan

${ }^{10}$ Department of Oncology, Immunology and Surgery, Nagoya City University Graduate School of Medical Sciences, Nagoya, Japan

*Corresponding author: Hiroko Yamashita

Breast Surgery, Hokkaido University Hospital, Kita 14, Nishi 5, Kita-ku, Sapporo 060-8648, Japan

Tel: +81-11-706-7381, Fax: +81-11-706-7384

E-mail: hirokoy@huhp.hokudai.ac.jp

Key words: Breast cancer, Post-relapse survival, Prognosis, Estrogen receptor-positive, Endocrine therapy 


\section{Abstract}

Background: Few studies have been performed on post-relapse survival in patients with early and late distant recurrence in estrogen receptor (ER)-positive, HER2-negative breast cancer.

Methods: A total of 205 patients with early distant recurrence and 134 patients with late distant recurrence of ER-positive, HER2-negative breast cancer who had undergone breast surgery or neoadjuvant chemotherapy between January 2000 and December 2004 were registered from nine institutions. Prognostic factors for post-relapse survival in patients with early and late recurrence were analyzed.

Results: Post-relapse survival was significantly longer in patients with late recurrence than in patients with early recurrence. Predictive factors for post-relapse survival in patients with early recurrence were lack of adjuvant chemotherapy, a long disease-free interval, and long durations of endocrine therapies and chemotherapies after relapse. In patients with late recurrence, post-relapse survival was significantly improved for those individuals with one metastatic organ at relapse and individuals who were treated with first-line and subsequent endocrine therapies for prolonged periods. Moreover, ER expression in primary breast tumors of late recurrence patients was significantly higher with a duration of first-line endocrine therapy $>6$ months than in those with a duration $\leq 6$ months.

Conclusion: Predictors for prognosis after relapse differed between patients with early and late distant recurrence. Endocrine responsiveness after relapse is a key factor for improved post-relapse survival, and it is thus important to establish whether metastatic tumors are 
endocrine-resistant in ER-positive, HER2-negative recurrent breast cancer.

\section{Abbreviations}

ER, estrogen receptor; PgR, progesterone receptor; HER2, human epidermal growth factor receptor type 2; Ki67 LI, Ki67 labeling index; IHC, immunohistochemistry 


\section{Introduction}

Women with estrogen receptor (ER)-positive breast cancer remain at particular risk for late recurrence, which is defined as relapse more than five years after initial treatment [1, 2]. Encouragingly, extended adjuvant endocrine therapy can reduce the frequency of late recurrence [3-6]. Accurate and reliable estimates of the risk of recurrence after five years of endocrine therapy, which would enable appropriate decisions regarding extended periods of this treatment modality, are therefore required. The risk of long-term relapse is related to the number of positive lymph nodes and the large size of invasive tumors [7-10]. Furthermore, multi-parametric molecular assays, including IHC4, OncotypeDX, EndoPredict, PAM50, and Breast Cancer Index, have been developed for predicting early (relapse less than five years after initial treatment) and/or late distant recurrence [8, 11].

Predictive factors for early and late recurrence, especially in postmenopausal ER-positive breast cancer, have previously been reported. However, there are few studies on predictors of prognosis after relapse. We previously demonstrated that high expression of ER, increased phosphorylation of ER $\alpha$ serine 167, and low expression of Ki67 and p53 in primary breast tumors were associated with response to first-line endocrine therapy and post-relapse survival in ER-positive metastatic breast cancer [12-15]. In these cases, tamoxifen was mainly used as an adjuvant endocrine therapy and/or as first-line endocrine therapy after relapse. However, prognostic factors after relapse might differ between women who receive adjuvant tamoxifen and those who are treated with aromatase inhibitors among postmenopausal women 
with ER-positive metastatic breast cancer. Furthermore, post-relapse survival might differ between patients with early or late recurrence. Recently, novel targeting agents such as everolimus, CDK 4/6 inhibitors, and PI3K inhibitors have been used in combination with endocrine therapies in ER-positive metastatic breast cancer [16, 17]. Therefore, understanding the response to endocrine therapy and survival in metastatic ER-positive breast cancer is critical in order to develop appropriate treatment strategies that utilize novel targeted agents [17].

We recently reported a multi-institutional joint retrospective study, carried out as Scientific Research of the Japanese Breast Cancer Society, using data from ER-positive, HER2-negative breast cancer patients with early and late distant recurrence [9]. Because all patients in the study were given their initial treatment between 2000 and 2004, almost 80\% of postmenopausal women received aromatase inhibitors as adjuvant endocrine therapy, and anthracyclins and/or taxanes were used as adjuvant or neoadjuvant chemotherapy. In the present study, which is a follow-up of the initial multi-institutional joint project, we analyzed factors that were prognostic for post-relapse survival in patients with early and late recurrence. 


\section{Patients and methods}

\section{Patients and breast cancer samples}

Patients with early and late distant recurrence of ER-positive, HER2-negative breast cancer who had undergone breast surgery or neoadjuvant chemotherapy between January 2000 and December 2004 were registered from nine institutions. They were incorporated into a multi-institutional joint study titled 'Analysis on biological characteristics and factors predicting late recurrence in breast cancer', and the project was carried out under the auspices of Scientific Research of the Japanese Breast Cancer Society [9]. Early recurrence was defined as relapse within 5 years, and late recurrence was defined as relapse more than 5 years after initial treatment. A total of 205 patients with early recurrence and 134 patients with late recurrence were analyzed in this study; 18 patients with early recurrence and 14 patients with late recurrence in the original cohort were excluded because information regarding first-line treatments after relapse was lacking. Treatment regimens and periods after the recurrence and post-relapse survival were retrospectively compared between the early and late recurrence groups. The study protocol was approved by the institutional review board in all nine institutions and conformed to the guidelines of the 1996 Declaration of Helsinki.

Expression of ER, progesterone receptor (PgR) and Ki67 in primary breast tumors was centrally assessed by immunohistochemistry (IHC). HER2-positive tumors were excluded from this study [9]. 


\section{Statistical analysis}

The chi-squared test, Student's $t$-test and the Mann-Whitney $U$-test were used to compare clinicopathological characteristics and treatments among patients with early and late recurrence. Estimation of post-relapse survival was performed using the Kaplan-Meier methods, and differences between survival curves were assessed with the log-rank test. Univariate and multivariate analyses with Cox proportional hazards regression models were used to identify independent prognostic factors in all patients. $p$ values $<0.05$ were considered significant. 


\section{Results}

\section{Comparison of clinicopathological characteristics and treatments between patients with} early and late recurrence

We collected data from a total of 205 women with early distant recurrence and 134 women with late distant recurrence in ER-positive, HER2-negative breast cancer (Table 1). The median disease-free intervals were 33 months (range 1-58 months) in the early recurrence cohort and 91 months (range 60-162 months) in the late recurrence cohort. Of the 43 (21\%) patients with early recurrence who did not receive adjuvant endocrine therapy, 12 individuals received adjuvant endocrine therapy for less than 6 months, and 4 patients relapsed before adjuvant endocrine therapy. Fifty-four women (26\%) with early relapse and 54 women $(40 \%)$ with late relapse had multiple metastatic organs at relapse $(p=0.009)$. In terms of metastatic sites at relapse, the proportion of lung metastases was significantly higher in patients with late relapse than in those with early relapse (35\% and $22 \%$, respectively, $p=$ 0.01). The fraction of patients who received endocrine therapy for the first-line treatment after relapse was $65 \%$ in the early recurrence cohort and $79 \%$ in the late recurrence cohort $(p=$ 0.03). There were no differences between the two cohorts with regard to time-to-progression with respect to first-line endocrine therapy (median 11 months, range 1-120 months versus median 12.5 months, range 0.6-77 months, early versus late relapse). On the other hand, total duration of endocrine therapies after relapse was significantly shorter in patients with early recurrence compared with those with late recurrence (median 14 months, range 0-120 months 
versus median 23 months, range $0-78$ months, early versus late relapse, $p=0.001$ ). The number of chemotherapy regimens was significantly higher in patients with early recurrence compared to those with late recurrence (median 3, range 1-8 versus median 2, range 1-6, early versus late relapse, $p=0.01$ ). The median follow-up periods after relapse were 76 months (range 14-177 months) in the early recurrence cohort and 133 months (range 66-175 months) in the late recurrence cohort. Post-relapse survival was significantly longer in patients with late recurrence than in patients with early recurrence ( $p=0.0074$, Fig. 1$)$. The 5-year post-relapse survivals were $35 \%$ in patients with early recurrence and $57 \%$ in patients with late recurrence.

\section{Factors predicting post-relapse survival in patients with early recurrence}

We then analyzed factors predicting post-relapse survival in patients with early recurrence. When analyzing post-relapse survival, setting the cutoff point at 3 years yielded the most significant difference between patient groups. Kaplan-Meier analysis showed that patients whose disease-free interval was more than 3 years displayed longer post-relapse survival compared with patients with shorter disease-free intervals ( $p=0.007$, Fig. 2 ). The 5-year post-relapse survivals were $29 \%$ in patients with a disease-free interval $\leq 3$ years and $49 \%$ in patients with a disease-free interval $>3$ years. Univariate analysis (Table 2) identified that significant correlations between long post-relapse survival and low tumor grade $(p=$ 0.0001), high PgR expression $(p=0.04)$, low Ki67 LI $(p=0.001)$, lack of adjuvant 
chemotherapy and radiation therapy ( $p=0.02$ and $p=0.02$, respectively), a long disease-free interval $(p=0.001)$, normal levels of tumor markers (CEA and/or CA15-3) at relapse $(p=$ 0.04), a long duration of first-line endocrine therapy after relapse $(p=0.01)$, and long durations of endocrine therapies and chemotherapies after relapse $(p<0.0001$ and $p<0.0001$, respectively). Receiving adjuvant endocrine therapy did not affect post-relapse survival. Multivariate analysis revealed significantly improved post-relapse survival in early recurrence patients with lack of adjuvant chemotherapy $(p=0.001)$, a long disease-free interval $(p=$ 0.002), and long durations of endocrine therapies and chemotherapies after relapse $(p<$ 0.0001 and $p<0.0001$, respectively) (Table 2).

\section{Factors predicting post-relapse survival in patients with late recurrence}

We next analyzed factors predicting post-relapse survival in patients with late recurrence. Kaplan-Meier analysis showed that patients who responded to first-line endocrine therapy (duration $>6$ months) had longer post-relapse survival compared with those on first-line endocrine therapy for $\leq 6$ months $(p<0.0001$, Fig. 3). Five-year post-relapse survival was $41 \%$ in patients with a duration of first-line endocrine therapy $\leq 6$ months and $70 \%$ in patients with a duration of first-line endocrine therapy $>6$ months. Univariate analysis (Table 3) identified significant correlations between long post-relapse survival and small number of positive lymph nodes $(p=0.049)$, use of radiation therapy, a single metastatic organ at relapse $(p=0.002)$, a long duration of first-line endocrine therapy $(p<0.0001)$, and a 
long total duration of endocrine therapies $(p=0.04)$ after relapse. Multivariate analysis revealed significantly improved post-relapse survival in late recurrence patients with a single metastatic organ at relapse $(p=0.03)$, a long duration of first-line endocrine therapy $(p<$ $0.0001)$ and a long total duration of endocrine therapies ( $p=0.01)$ after relapse (Table 3).

\section{Comparison of clinicopathological characteristics and treatments according to response to first-line endocrine therapy after relapse in patients with late recurrence}

The above results indicate that duration of first-line endocrine therapy was the most significant prognostic factor for post-relapse survival in patients with late recurrence. Therefore, we compared the clinicopathological characteristics and treatments between a duration of first-line endocrine therapy $\leq 6$ months and $>6$ months in patients who were treated endocrine therapy as the initial treatment at relapse (Table 4). ER expression in primary breast tumors was significantly higher in patients with a duration of first-line endocrine therapy $>6$ months than in those with a duration $\leq 6$ months $(p=0.03)$. The mean percentage of ER positive cells was $66.0 \%$ in patients with a duration of first-line endocrine therapy $>6$ months, and $51.6 \%$ in those with a duration $\leq 6$ months (Table 4 ). Moreover, the total duration of endocrine therapies after relapse was significantly longer in patients with a duration of first-line endocrine therapy $>6$ months $(p<0.0001)$. Mean total duration of endocrine therapies after relapse was 32.7 months in patients that were treated with first-line endocrine therapy for $>6$ months, while it was 12.0 months in those with a duration of $\leq 6$ 
months (Table 4). Other clinicopathological factors and treatments were similar, -in patients with late recurrence independently of the duration of first-line endocrine therapy. 


\section{Discussion}

We investigated the clinicopathological factors and treatments predicting post-relapse survival in ER-positive, HER2-negative breast cancer patients with early and late recurrence. Our results demonstrated that post-relapse survival was significantly longer in patients with late recurrence than in those with early recurrence, and that predictors for prognosis after relapse were different between the two cohorts. Predictive factors for post-relapse survival in patients with early recurrence were lack of adjuvant chemotherapy, a long disease-free interval, and long durations of endocrine and chemotherapies after relapse. In patients with late recurrence, the involvement of metastasis in only one organ at relapse, a long duration of first-line endocrine therapy, and a long total duration of endocrine therapies after relapse significantly improved post-relapse survival. Moreover, ER expression in primary breast tumors of late recurrence patients was significantly higher in patients with a duration of first-line endocrine therapy $>6$ months than in those with a duration $\leq 6$ months.

Our previous study demonstrated that predictive factors for early and late recurrence were mainly a large tumor size and a large number of positive lymph nodes [9]. However, as expected, tumor size and lymph node status at the initial treatments were not associated with post-relapse survival in patients with both early and late recurrence. Approximately two-thirds of women who relapsed within 5 years had received anthracyclines and/or taxanes as adjuvant or neoadjuvant chemotherapy. Moreover, approximately $90 \%$ of patients with early recurrence developed distant metastasis during adjuvant endocrine therapy. Therefore, we infer that most 
patients with early recurrence in our study were resistant to standard endocrine therapies and chemotherapies. On the other hand, our present study shows that lack of adjuvant chemotherapy, a long disease-free interval, and long durations of endocrine and chemotherapies after relapse were independent prognostic factors for patients with early relapse; furthermore, low tumor grade and low Ki67 LI were associated with longer post-relapse survival in univariate analysis. The 5-year post-relapse survival was significantly longer in patients with a disease-free interval $>3$ years than in patients with a disease-free interval $\leq 3$ years. Despite the occurrence of relapse within 5 years of the initial treatments, we suggest that patients with a disease-free interval $>3$ years have relatively low-grade tumors that essentially remain responsive to other endocrine or chemotherapeutic agents.

Our results further show that single metastatic organ at relapse, a long duration of first-line endocrine therapy and a long total duration of endocrine therapies after relapse are independent prognostic factors for post-relapse survival in patients with late recurrence. Breast cancer patients with bone-only metastases had excellent clinical outcomes, especially in hormone receptor-positive breast cancer, and the median post-relapse survival was 79.4 months [18]. Endocrine therapy is usually recommended as first-line treatment for patients with bone-only metastases in ER-positive breast cancer. Here, we demonstrated that $79 \%$ of patients with late relapse received endocrine therapy as first-line treatment after relapse. Moreover, $76 \%$ of patients who received endocrine therapy as first-line treatment responded to the treatment, such as a duration of first-line endocrine therapy $>6$ months. In contrast, 
$24 \%$ of patients who did not respond to first-line endocrine therapy had a worse prognosis, as the 5 -year post-relapse survival was $41 \%$; this is similar to the 5 -year post-relapse survival in patients with early recurrence (35\%). Most patients who do not respond to the first-line endocrine therapy after relapse are likely to be endocrine-resistant, and chemotherapies are usually selected as the second-line treatment [19].

Our results show that in patients with late recurrence, ER expression in primary breast tumors was significantly higher in patients with a duration of first-line endocrine therapy $>6$ months than in those treated for shorter periods, whereas other clinicopathological factors were not correlated with response to first-line endocrine therapy. Although ER expression and endocrine responsiveness in metastatic sites are reduced as the disease progresses [20], malignancies at the initial metastatic sites may retain the biology of primary breast tumors, despite the lapse of time since the initial treatments [21]. Furthermore, a recent study showed that an epithelial to mesenchymal transition-related gene signature in the primary breast tumors is related stromal activation and escape from disease dormancy [22].

Recently, inhibitors of mTOR, CDK 4/6, and PI3K have been developed and used in combination with endocrine therapies in ER-positive metastatic breast cancer [16]. Understanding predictors for the response to endocrine therapy and overall survival in metastatic ER-positive breast cancer will facilitate the stratification of patients to be treated with specific targeted molecular therapies.

In conclusion, our study demonstrates that the predictors of prognosis after relapse 
are different between patients with early and late distant recurrence. Endocrine responsiveness after relapse is a key factor for improved post-relapse survival, and it is thus important to establish whether metastatic tumors are endocrine-resistant in ER-positive, HER2-negative recurrent breast cancer. 


\section{Acknowledgments}

This study was supported by Grant-in-Aid for Scientific Research from the Japanese

Breast Cancer Society.

\section{Conflict of interest statement}

The authors have no conflict of interest. 


\section{References}

1. Jatoi I, Anderson WF, Jeong JH, Redmond CK. Breast cancer adjuvant therapy: time to consider its time-dependent effects. J Clin Oncol 2011;29:2301-4.

2. Saphner T, Tormey DC, Gray R. Annual hazard rates of recurrence for breast cancer after primary therapy. J Clin Oncol 1996;14:2738-46.

3. Goss PE, Ingle JN, Pater JL, Martino S, Robert NJ, Muss HB, et al. Late extended adjuvant treatment with letrozole improves outcome in women with early-stage breast cancer who complete 5 years of tamoxifen. J Clin Oncol 2008;26:1948-55.

4. Jakesz R, Greil R, Gnant M, Schmid M, Kwasny W, Kubista E, et al. Extended adjuvant therapy with anastrozole among postmenopausal breast cancer patients: results from the randomized Austrian Breast and Colorectal Cancer Study Group Trial 6a. J Natl Cancer Inst 2007;99:1845-53.

5. Davies C, Pan H, Godwin J, Gray R, Arriagada R, Raina V, et al. Long-term effects of continuing adjuvant tamoxifen to 10 years versus stopping at 5 years after diagnosis of oestrogen receptor-positive breast cancer: ATLAS, a randomised trial. Lancet 2013;381:805-16.

6. Zdenkowski N, Forbes JF, Boyle FM, Kannourakis G, Gill PG, Bayliss E, et al. Observation versus late reintroduction of letrozole as adjuvant endocrine therapy for hormone receptor-positive breast cancer (ANZ0501 LATER): an open-label randomised, controlled trial. Ann Oncol 2016;27:806-12.

7. Kennecke HF, Olivotto IA, Speers C, Norris B, Chia SK, Bryce C, et al. Late risk of relapse and mortality among postmenopausal women with estrogen responsive early breast cancer after 5 years of tamoxifen. Ann Oncol 2007;18:45-51.

8. Sestak I, Cuzick J. Markers for the identification of late breast cancer recurrence. Breast Cancer Res 2015;17:10.

9. Yamashita H, Ogiya A, Shien T, Horimoto Y, Masuda N, Inao T, et al. Clinicopathological factors predicting early and late distant recurrence in estrogen receptor-positive, HER2-negative breast cancer. Breast Cancer 2015. doi:10.1007/s12282-015-0649-0.

10. Ahn SG, Lee HM, Cho SH, Bae SJ, Lee SA, Hwang SH, et al. The difference in prognostic factors between early recurrence and late recurrence in estrogen receptor-positive breast cancer: nodal stage differently impacts early and late recurrence. PLoS One 2013;8:e63510.

11. Hayes DF. Clinical utility of genetic signatures in selecting adjuvant treatment: Risk stratification for early vs. late recurrences. Breast 2015;24 Suppl 2:S6-S10.

12. Yamashita H, Ando $\mathrm{Y}$, Nishio $M$, Zhang Z, Hamaguchi M, Mita K, et al. Immunohistochemical evaluation of hormone receptor status for predicting response to endocrine therapy in metastatic breast cancer. Breast Cancer 2006;13:74-83.

13. Endo Y, Toyama T, Takahashi S, Sugiura H, Yoshimoto N, Iwasa M, et al. High estrogen receptor expression and low Ki67 expression are associated with improved time to 
progression during first-line endocrine therapy with aromatase inhibitors in breast cancer. Int J Clin Oncol 2011;16:512-8.

14. Yamashita H, Nishio M, Kobayashi S, Ando Y, Sugiura H, Zhang Z, et al. Phosphorylation of estrogen receptor alpha serine 167 is predictive of response to endocrine therapy and increases postrelapse survival in metastatic breast cancer. Breast Cancer Res 2005;7:R753-64.

15. Yamashita H, Toyama T, Nishio M, Ando Y, Hamaguchi M, Zhang Z, et al. p53 protein accumulation predicts resistance to endocrine therapy and decreased post-relapse survival in metastatic breast cancer. Breast Cancer Res 2006;8:R48.

16. Johnston SR. Enhancing Endocrine Therapy for Hormone Receptor-Positive Advanced Breast Cancer: Cotargeting Signaling Pathways. J Natl Cancer Inst 2015;107:

17. Perez EA. Treatment strategies for advanced hormone receptor-positive and human epidermal growth factor 2-negative breast cancer: the role of treatment order. Drug Resist Updat 2016;24:13-22.

18. Lee SJ, Park S, Ahn HK, Yi JH, Cho EY, Sun JM, et al. Implications of bone-only metastases in breast cancer: favorable preference with excellent outcomes of hormone receptor positive breast cancer. Cancer Res Treat 2011;43:89-95.

19. Hortobagyi GN. Treatment of breast cancer. N Engl J Med 1998;339:974-84.

20. Jeselsohn R, Buchwalter G, De Angelis C, Brown M, Schiff R. ESR1 mutations-a mechanism for acquired endocrine resistance in breast cancer. Nat Rev Clin Oncol 2015;12:573-83.

21. Zhang XH, Giuliano M, Trivedi MV, Schiff R, Osborne CK. Metastasis dormancy in estrogen receptor-positive breast cancer. Clin Cancer Res 2013;19:6389-97.

22. Cheng Q, Chang JT, Gwin WR, Zhu J, Ambs S, Geradts J, et al. A signature of epithelial-mesenchymal plasticity and stromal activation in primary tumor modulates late recurrence in breast cancer independent of disease subtype. Breast Cancer Res 2014;16:407. 


\section{Figure legends}

Fig. 1: Post-relapse survival of patients with early and late distant recurrence. The 5-year post-relapse survivals were $35 \%$ in patients with early recurrence and $57 \%$ in those with late recurrence $(p=0.0074)$.

Fig. 2: Post-relapse survival according to disease-free interval in patients with early recurrence. The 5-year post-relapse survivals were $29 \%$ in patients with a disease-free interval $\leq 3$ years and $49 \%$ in patients with a disease-free interval $>3$ years $(p=0.007)$.

Fig. 3: Post-relapse survival according to the duration of first-line endocrine therapy in patients with late recurrence. The 5-year post-relapse survivals were $41 \%$ in patients with a duration of first-line endocrine therapy $\leq 6$ months and $70 \%$ in patients with a duration of first-line endocrine therapy $>6$ months $(p<0.0001)$. 
Fig. 1

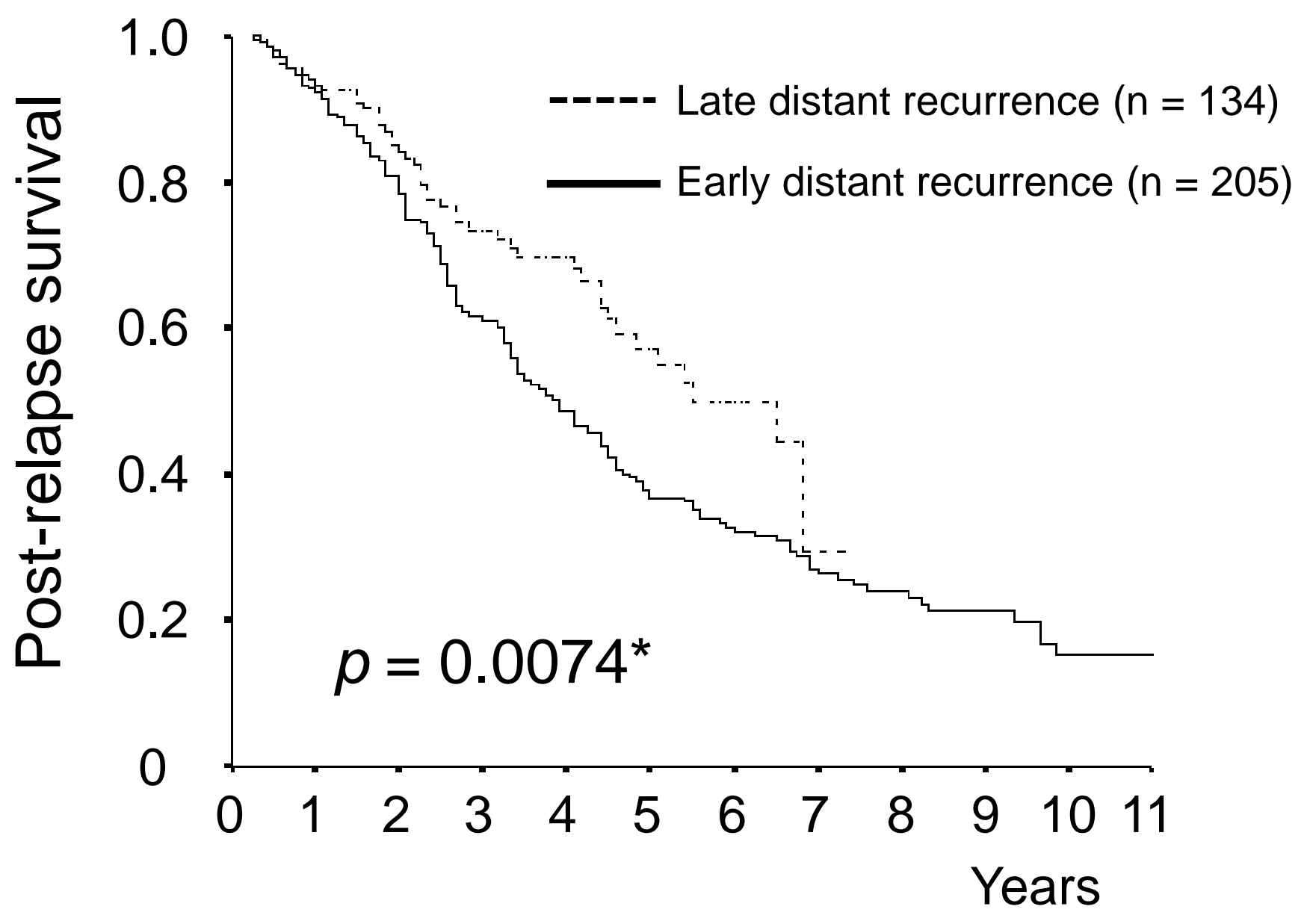


Fig. 2

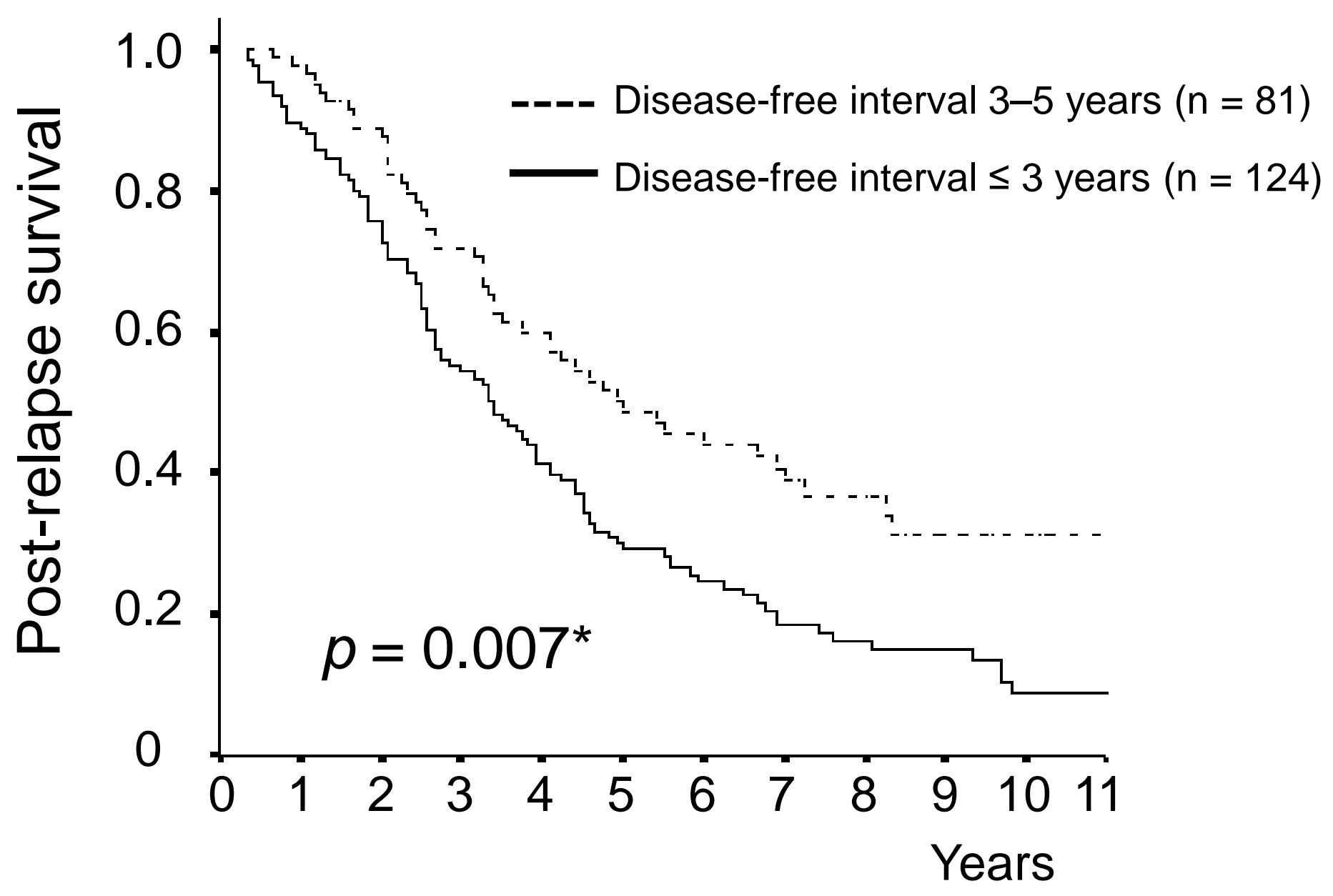




\section{Fig. 3}

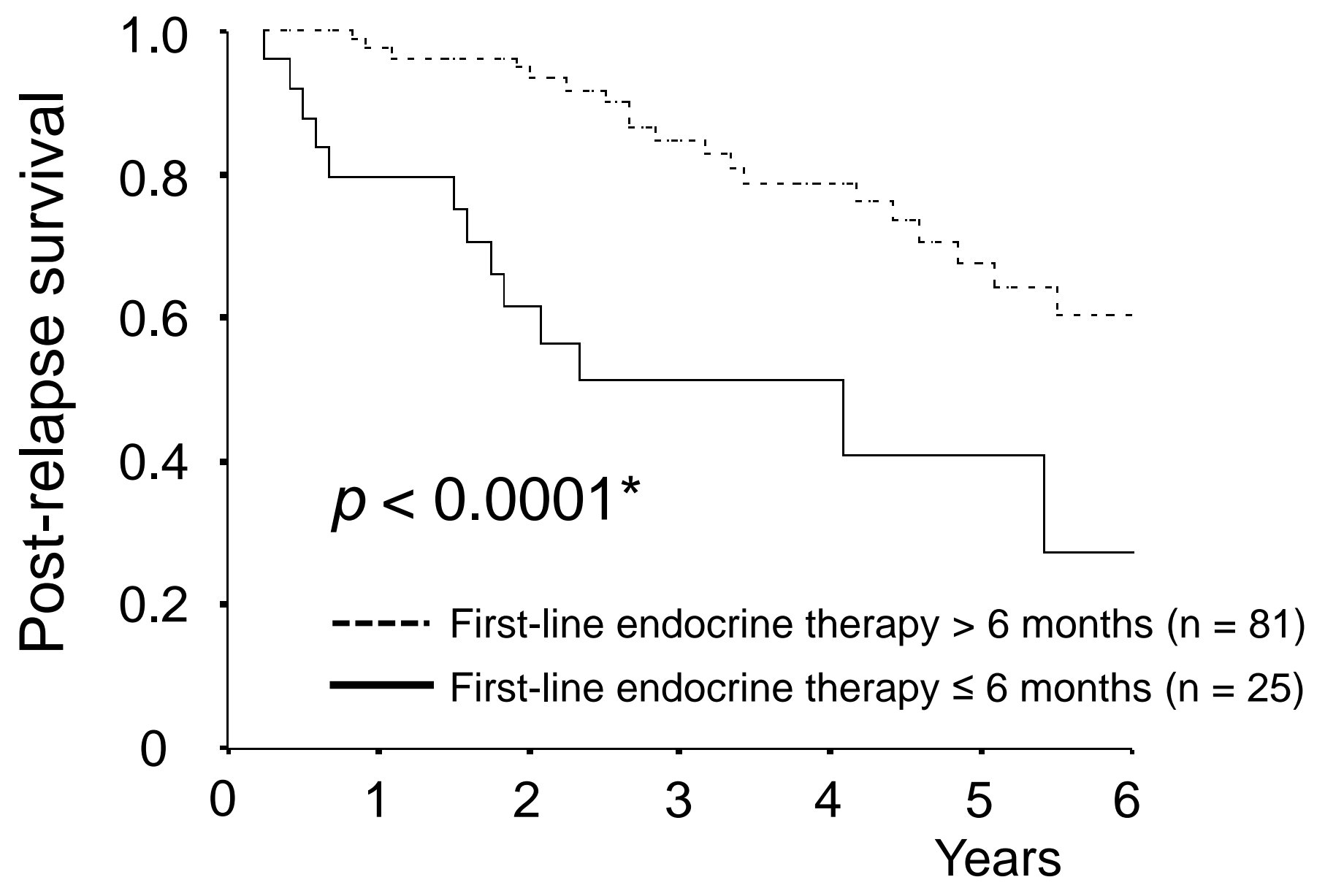


Table 1. Comparison of clinicopathological characteristics and treatments between patients with early and late recurrence

\begin{tabular}{|c|c|c|c|}
\hline & $\begin{array}{c}\text { Early recurrence } \\
(\mathrm{n}=205)\end{array}$ & $\begin{array}{c}\text { Late recurrence } \\
(\mathrm{n}=134)\end{array}$ & $P$-value \\
\hline \multicolumn{4}{|c|}{ Menopausal status (at the time of the initial therapy) } \\
\hline Premenopausal & $104(51 \%)$ & $55(41 \%)$ & \multirow{2}{*}{0.08} \\
\hline Postmenopausal & $101(49 \%)$ & 79 (59\%) & \\
\hline \multicolumn{4}{|l|}{ Tumor category (pathological) } \\
\hline pT1 & $60(29 \%)$ & $58(43 \%)$ & \multirow{5}{*}{$0.03 *$} \\
\hline pT2 & $100(49 \%)$ & $61(46 \%)$ & \\
\hline pT3 & $34(17 \%)$ & $9(7 \%)$ & \\
\hline pT4 & $8(4 \%)$ & $4(3 \%)$ & \\
\hline Unknown & $3(2 \%)$ & $2(2 \%)$ & \\
\hline \multicolumn{4}{|l|}{ No. of positive lymph nodes } \\
\hline 0 & $46(22 \%)$ & $50(37 \%)$ & \multirow{5}{*}{$<0.0001^{*}$} \\
\hline $1-3$ & $64(31 \%)$ & $54(40 \%)$ & \\
\hline $4-9$ & $38(19 \%)$ & $13(10 \%)$ & \\
\hline$\geq 10$ & $55(27 \%)$ & $17(13 \%)$ & \\
\hline Unknown & $2(1 \%)$ & 0 & \\
\hline \multicolumn{4}{|l|}{ Histological type } \\
\hline Invasive ductal carcinoma & 192 (94\%) & $126(93 \%)$ & \multirow{3}{*}{$0.03 *$} \\
\hline Invasive lobular carcinoma & $12(6 \%)$ & $3(2 \%)$ & \\
\hline Other & $1(0.5 \%)$ & $5(6 \%)$ & \\
\hline \multicolumn{4}{|l|}{ Tumor grade } \\
\hline 1 & $60(29 \%)$ & $54(40 \%)$ & \multirow{3}{*}{0.09} \\
\hline 2 & $90(44 \%)$ & $53(40 \%)$ & \\
\hline 3 & $55(27 \%)$ & 27 (20\%) & \\
\hline ER (\%), median (range) & $70.0(1-100)$ & $70.0(1-100)$ & 0.71 \\
\hline PgR (\%), median (range) & $30.0(0-100)$ & $30.0(1-100)$ & 0.45 \\
\hline Ki67 LI (\%), median (range) & $14.0(0-53.0)$ & $9.2(0-70.0)$ & $0.01 *$ \\
\hline \multicolumn{4}{|l|}{ Neoadjuvant chemotherapy } \\
\hline Yes & $48(23 \%)$ & $14(10 \%)$ & \multirow{2}{*}{$0.002 *$} \\
\hline No & 157 (77\%) & $120(90 \%)$ & \\
\hline \multicolumn{4}{|l|}{ Adjuvant therapy } \\
\hline \multicolumn{4}{|l|}{ Endocrine therapy } \\
\hline Yes & 162 (79\%) & 122 (91\%) & \multirow{3}{*}{$0.001^{*}$} \\
\hline No & $43(21 \%)$ & $10(8 \%)$ & \\
\hline Unknown & 0 & $2(2 \%)$ & \\
\hline \multicolumn{4}{|l|}{ Chemotherapy } \\
\hline Yes & $126(62 \%)$ & $76(57 \%)$ & \multirow{3}{*}{0.47} \\
\hline No & $78(38 \%)$ & $58(43 \%)$ & \\
\hline Unknown & $1(0.5 \%)$ & 0 & \\
\hline \multicolumn{4}{|l|}{ Radiotherapy } \\
\hline Yes & $98(48 \%)$ & $45(34 \%)$ & \multirow{3}{*}{$0.03 *$} \\
\hline No & 105 (51\%) & $88(66 \%)$ & \\
\hline Unknown & $2(1 \%)$ & $1(1 \%)$ & \\
\hline
\end{tabular}


Elevation of serum levels of tumor markers at relapse

No elevation

CEA only

CA15-3 only

Both CEA and CA15-3

Unknown

No. of metastatic organs at relapse

Single

Multiple

Metastatic site at relapse

Bone

Liver

Lung

Brain

Other

First-line treatment after relapse

Endocrine therapy

Chemotherapy

Other

None

Time-to-progression of first-line endocrine therapy (months)
88 (43\%)

$24(12 \%)$

$41(20 \%)$

$45(22 \%)$

$7(3 \%)$

$151(74 \%)$

$54(26 \%)$

$101(49 \%)$

$42(20 \%)$

$46(22 \%)$

$3(1 \%)$

$46(22 \%)$

$134(65 \%)$

$63(31 \%)$

$5(2 \%)$

$3(2 \%)$

$11(1-120)$

Total duration of endocrine therapies after relapse (months) median (range)

$14(0-120)$

No. of chemotherapy regimens after relapse median (range)

$3(1-8)$

Total duration of chemotherapies after relapse (months) median (range)

$19(2-126)$

$59(44 \%)$

$27(20 \%)$

$20(15 \%)$

0.17

$26(20 \%)$

$2(2 \%)$

$80(60 \%)$

$54(40 \%)$

$0.009 *$

$63(47 \%) \quad 0.69$

$20(15 \%) \quad 0.20$

47 (35\%) $\quad 0.01 *$

$2(1 \%) \quad 0.98$

$29(22 \%) \quad 0.86$

$106(79 \%)$

$26(19 \%)$

0

$0.03^{*}$

$2(2 \%)$

$12.5(0.6-77) \quad 0.41$

$23(0-78) \quad 0.001^{*}$

$2(1-6) \quad 0.01 *$

$18.5(2-59) \quad 0.12$

${ }^{*} p<0.05$ is considered significant. 
Table 2. Univariate and multivariate analyses of factors predicting post-relapse survival in patients with early recurrence

\begin{tabular}{|c|c|c|c|c|c|c|}
\hline & \multicolumn{3}{|c|}{ Univariate } & \multicolumn{3}{|c|}{ Multivariate } \\
\hline & HR & $95 \%$ CI & $P$-value & HR & $95 \% \mathrm{CI}$ & $P$-value \\
\hline Menopausal status & 1.278 & $0.928-1.761$ & 0.13 & & & \\
\hline Tumor category (pathological) & 0.999 & 0.998-1.001 & 0.43 & & & \\
\hline No. of positive lymph nodes & 1.109 & 0.997-1.001 & 0.47 & & & \\
\hline Histological type & 1.806 & 0.915-3.565 & 0.09 & & & \\
\hline Tumor grade & 1.463 & $1.173-1.824$ & $0.001 *$ & 1.293 & $0.940-1.780$ & 0.11 \\
\hline ER status (\%) & 1.000 & 0.995-1.005 & 0.99 & & & \\
\hline PgR status (\%) & 0.995 & 0.990-1.000 & $0.04 *$ & 1.002 & 0.995-1.009 & 0.56 \\
\hline Ki67 LI (\%) & 1.024 & 1.010-1.038 & $0.001 *$ & 1.001 & $0.980-1.022$ & 0.94 \\
\hline Neoadjuvant chemotherapy & 1.011 & $0.695-1.470$ & 0.96 & & & \\
\hline \multicolumn{7}{|l|}{ Adjuvant therapy } \\
\hline Endocrine therapy & 0.873 & 0.595-1.279 & 0.49 & & & \\
\hline Chemotherapy & 1.002 & $1.000-1.004$ & $0.02 *$ & 2.508 & $1.455-4.325$ & $0.001^{*}$ \\
\hline Radiotherapy & 1.002 & $1.000-1.003$ & $0.02 *$ & 1.000 & 0.997-1.002 & 0.77 \\
\hline Disease-free interval & 0.982 & 0.972-0.993 & $0.001 *$ & 0.975 & 0.959-0.991 & $0.002 *$ \\
\hline No. of metastatic organs at relapse & 1.246 & $0.867-1.790$ & 0.23 & & & \\
\hline $\begin{array}{l}\text { Elevation of CEA and/or CA15-3 at } \\
\text { relapse }\end{array}$ & 1.001 & $1.000-1.002$ & $0.04 *$ & 1.000 & $1.000-1.003$ & 0.10 \\
\hline First-line treatment after relapse & 1.451 & 1.079-1.951 & $0.01 *$ & & & \\
\hline $\begin{array}{l}\text { Duration of first-line endocrine therapy } \\
\text { ( } \leq 6 \text { months vs. }>6 \text { months) }\end{array}$ & 0.588 & 0.384-0.900 & $0.01 *$ & 0.816 & $0.462-1.441$ & 0.48 \\
\hline $\begin{array}{l}\text { Total duration of endocrine therapies } \\
\text { after relapse (months) }\end{array}$ & 0.967 & $0.957-0.976$ & $<0.0001^{*}$ & 0.933 & $0.917-0.950$ & $<0.0001 *$ \\
\hline $\begin{array}{l}\text { No. of chemotherapy regimens after } \\
\text { relapse }\end{array}$ & 1.003 & 0.931-1.080 & 0.93 & & & \\
\hline $\begin{array}{l}\text { Total duration of chemotherapies after } \\
\text { relapse (months) }\end{array}$ & 0.982 & 0.974-0.989 & $<0.0001^{*}$ & 0.944 & 0.929-0.959 & $<0.0001^{*}$ \\
\hline
\end{tabular}

${ }^{*} p<0.05$ is considered significant. 
Table 3. Univariate and multivariate analyses of factors predicting post-relapse survival in patients with late recurrence

\begin{tabular}{|c|c|c|c|c|c|c|}
\hline & \multicolumn{3}{|c|}{ Univariate } & \multicolumn{3}{|c|}{ Multivariate } \\
\hline & HR & $95 \% \mathrm{CI}$ & $P$-value & HR & $95 \% \mathrm{CI}$ & $P$-value \\
\hline Menopausal status & 1.622 & $0.873-3.016$ & 0.13 & & & \\
\hline Tumor category (pathological) & 0.777 & $0.494-1.222$ & 0.27 & & & \\
\hline No. of positive lymph nodes & 1.333 & $1.002-1.774$ & $0.049^{*}$ & 1.212 & $0.849-1.730$ & 0.29 \\
\hline Histological type & 0.864 & $0.410-1.824$ & 0.70 & & & \\
\hline Tumor grade & 1.167 & $0.787-1.729$ & 0.44 & & & \\
\hline ER status (\%) & 1.003 & 0.994-1.013 & 0.45 & & & \\
\hline PgR status (\%) & 1.000 & 0.991-1.008 & 0.95 & & & \\
\hline Ki67 LI (\%) & 1.012 & $0.992-1.033$ & 0.24 & & & \\
\hline Neoadjuvant chemotherapy & 0.712 & $0.220-2.300$ & 0.57 & & & \\
\hline \multicolumn{7}{|l|}{ Adjuvant therapy } \\
\hline Endocrine therapy & 0.863 & $0.339-2.196$ & 0.76 & & & \\
\hline Chemotherapy & 1.014 & $0.559-1.839$ & 0.96 & & & \\
\hline Radiotherapy & 1.002 & $1.000-1.004$ & $0.04 *$ & 1.025 & $0.487-2.153$ & 0.95 \\
\hline Disease-free interval & 0.991 & $0.944-1.006$ & 0.23 & & & \\
\hline $\begin{array}{l}\text { No. of metastatic organs at relapse } \\
\text { (Single vs. multiple) }\end{array}$ & 2.570 & $1.413-4.676$ & $0.002 *$ & 2.185 & $1.076-4.438$ & $0.03 *$ \\
\hline $\begin{array}{l}\text { Elevation of CEA and/or CA15-3 at } \\
\text { relapse }\end{array}$ & 0.997 & $0.985-1.010$ & 0.68 & & & \\
\hline First-line treatment after relapse & 1.580 & $0.815-3.064$ & 0.18 & & & \\
\hline $\begin{array}{l}\text { Duration of first-line endocrine therapy } \\
\text { ( } \leq 6 \text { months vs. }>6 \text { months })\end{array}$ & 0.858 & $0.800-0.921$ & $<0.0001^{*}$ & 0.252 & $0.119-0.534$ & $<0.0001^{*}$ \\
\hline $\begin{array}{l}\text { Total duration of endocrine therapies } \\
\text { after relapse (months) }\end{array}$ & 1.014 & $1.000-1.028$ & $0.04 *$ & 1.021 & $1.004-1.038$ & $0.01 *$ \\
\hline $\begin{array}{l}\text { No. of chemotherapy regimens after } \\
\text { relapse }\end{array}$ & 1.077 & $0.925-1.254$ & 0.34 & & & \\
\hline $\begin{array}{l}\text { Total duration of chemotherapies after } \\
\text { relapse (months) }\end{array}$ & 0.986 & & 0.19 & & & \\
\hline
\end{tabular}

${ }^{*} p<0.05$ is considered significant. 
Table 4. Comparison of clinicopathological characteristics and treatments according to response to first-line endocrine therapy after relapse in patients with late recurrence

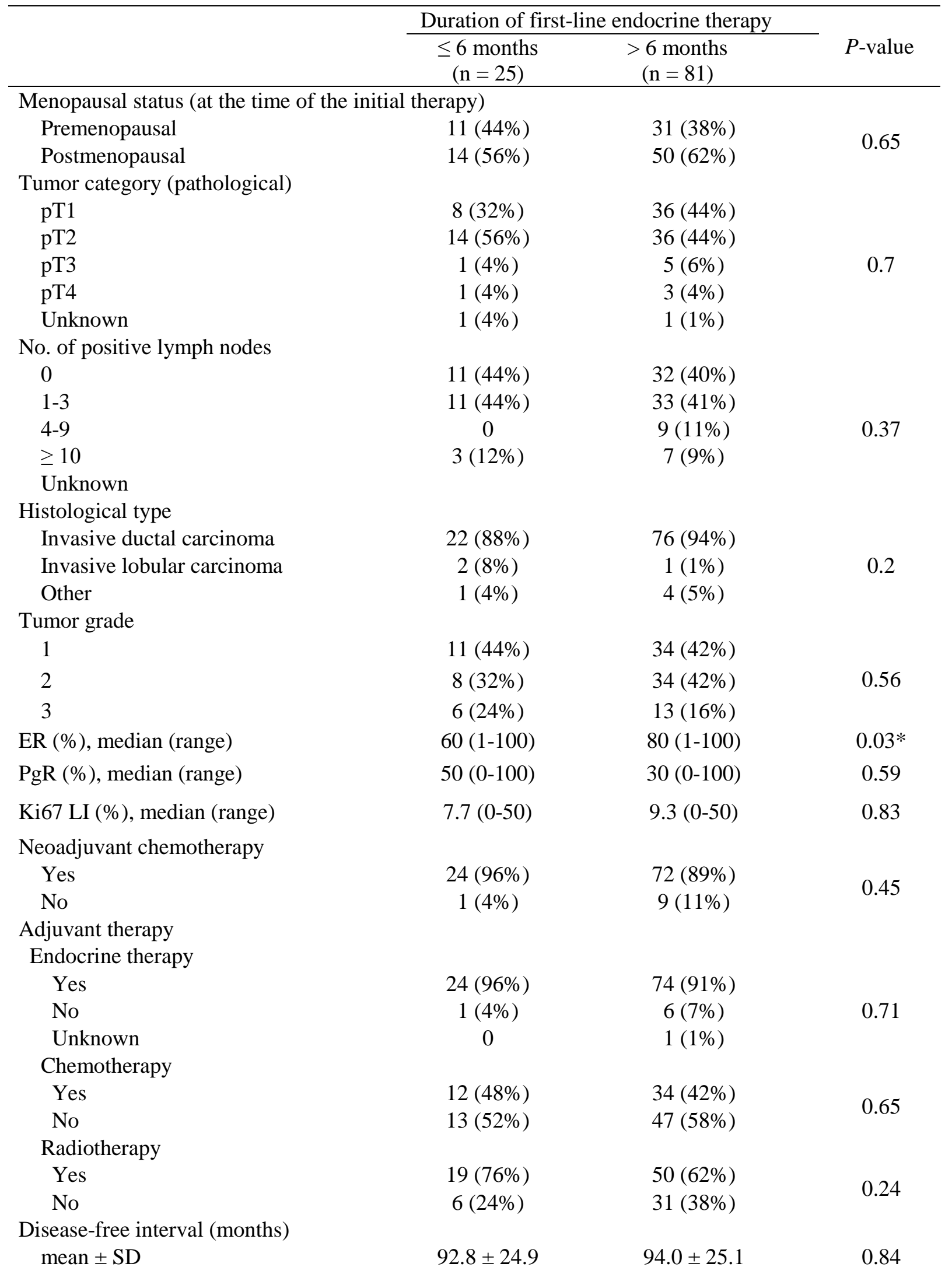


Elevation of CEA and/or CA15-3 at relapse

No elevation

CEA only

CA15-3 only

Both CEA and CA15-3

Unknown

No. of metastatic organs at relapse

Single

Multiple

First-line endocrine therapy

Aromatase inhibitors

SERM

Fulvestrant

LHRH agonist

Total duration of endocrine therapies after relapse (months) median (range)

No. of chemotherapy regimens after relapse median (range)

Total duration of chemotherapies after relapse (months)

median (range)

${ }^{*} p<0.05$ is considered significant.
$18(72 \%)$

$6(24 \%)$

$1(4 \%)$

0

$6(1-63)$

$1(0-5)$

$4(0-39)$
$42(52 \%)$

$15(19 \%)$

$9(11 \%)$

0.16

$13(16 \%)$

$2(3 \%)$

$61(75 \%)$

$17(21 \%)$

$2(3 \%)$

$1(1 \%)$

$29.5(7-78)$

$<0.0001^{*}$

$0(0-6)$

0.06

$0(0-49)$

0.16

.

$\begin{array}{lll}15(60 \%) & 54(67 \%) & 0.68 \\ 10(40 \%) & 27(33 \%) & \end{array}$

$\begin{array}{lll}15(60 \%) & 54(67 \%) & 0.68 \\ 10(40 \%) & 27(33 \%) & \end{array}$

$\begin{array}{lll}15(60 \%) & 54(67 \%) & 0.68 \\ 10(40 \%) & 27(33 \%) & \end{array}$

$\begin{array}{ccc}5(20 \%) & 15(19 \%) & \\ 5(20 \%) & 9(11 \%) & 0.16 \\ 8(32 \%) & 13(16 \%) & \\ 0 & 2(3 \%) & \end{array}$

(

